\title{
Systematizing Confidence in Open Research and Evidence (SCORE)
}

\author{
SCORE Collaboration ${ }^{1}$
}

\begin{abstract}
Assessing the credibility of research claims is a central, continuous, and laborious part of the scientific process. Credibility assessment strategies range from expert judgment to aggregating existing evidence to systematic replication efforts. Such assessments can require substantial time and effort. Research progress could be accelerated if there were rapid, scalable, accurate credibility indicators to guide attention and resource allocation for further assessment. The SCORE program is creating and validating algorithms to provide confidence scores for research claims at scale. To investigate the viability of scalable tools, teams are creating: a database of claims from papers in the social and behavioral sciences; expert and machine generated estimates of credibility; and, evidence of reproducibility, robustness, and replicability to validate the estimates. Beyond the primary research objective, the data and artifacts generated from this program will be openly shared and provide an unprecedented opportunity to examine research credibility and evidence.
\end{abstract}

\section{Words}

Keywords: Metascience, replicability, reproducibility, social sciences, credibility, algorithms

\footnotetext{
${ }^{1}$ Co-authors listed alphabetically: Nazanin Alipourfard, University of Southern California ; Beatrix Arendt, Center for Open Science ; Daniel Benjamin , University of Southern California ; Noam Benkler, SIFT ; Mark Burstein , SIFT ; Martin Bush, University of Melbourne ; James Caverlee , Texas A\&M University ; Yiling Chen, Harvard University ; Chae Clark, TwoSix Technologies ; Anna Dreber, Stockholm School of Economics ; Timothy M. Errington, Center for Open Science ; Fiona Fidler , University of Melbourne ; Nicholas Fox, Center for Open Science ; Aaron Frank , RAND Corporation ; Hannah Fraser , University of Melbourne ; Scott Friedman , SIFT ; Ben Gelman , TwoSix Technologies ; James Gentile, TwoSix Technologies ; C Lee Giles, The Pennsylvania State University ; Michael Gordon, Massey University; Reed Gordon-Sarney, TwoSix Technologies ; Christopher Griffin , The Pennsylvania State University ; Timothy Gulden, RAND Corporation ; Krystal Hahn, Center for Open Science ; Robert Hartman , The MITRE Corporation ; Felix Holzmeister , University of Innsbruck ; Xia Hu , Texas A\&M University ; Magnus Johannesson , Stockholm School of Economics ; Lee Kezar , University of Southern California ; Melissa Kline Struhl , Center for Open Science ; Ugur Kuter, SIFT ; Anthony Kwasnica, The Pennsylvania State University ; Dong-Ho Lee , University of Southern California ; Kristina Lerman , University of Southern California ; Yang Liu , University of California, Santa Cruz ; Zach Loomas , Center for Open Science ; Bri Luis , Center for Open Science ; Ian Magnusson, SIFT ; Michael Bishop, Ottawa, ON, Canada ; Olivia Miske, Center for Open Science ; Fallon Mody , University of Melbourne ; Fred Morstatter , University of Southern California ; Brian A. Nosek, Center for Open Science; University of Virginia ; E. Simon Parsons, Center for Open Science ; David Pennock, Rutgers University ; Thomas Pfeiffer, Massey University; Haochen Pi , University of Southern California ; Jay Pujara, University of Southern California ; Sarah Rajtmajer, The Pennsylvania State University ; Xiang Ren , University of Southern California ; Abel Salinas , University of Southern California ; Ravi Selvam , University of Southern California ; Frank Shipman , Texas A\&M University ; Priya Silverstein , Center for Open Science; Institute for Globally Distributed Open Research and Education ; Amber Sprenger, The MITRE Corporation ; Anna Squicciarini, The Pennsylvania State University ; Stephen Stratman, The MITRE Corporation ; Kexuan Sun , University of Southern California ; Saatvik Tikoo, University of Southern California ; Charles R. Twardy, Jacobs / George Mason ; Andrew Tyner , Center for Open Science ; Domenico Viganola , World Bank ; Juntao Wang, Harvard University ; David Wilkinson , University of Melbourne ; Bonnie Wintle , University of Melbourne ; Jian Wu , Old Dominion University
} 
A primary activity of science is evaluating the credibility of claims--assertions reported as findings from the evaluation of evidence. Researchers create evidence and make claims about what that evidence means. Others assess those claims to determine their credibility including evaluating reliability, validity, generalizability, and applicability. Assessment occurs by journal reviewers during the peer review process; by readers deciding whether claims should inform their judgment; by researchers trying to replicate, extend, confirm, or challenge prior claims; by funders deciding what is worth further investment; and by practitioners and policymakers determining whether the claims should inform policy or practice.

Assessing confidence in research claims is important and resource intensive. A reader must read and think about a paper to assess confidence in its claims using their expert judgment. $A$ researcher expends substantial effort planning, conducting, and reporting follow up research to assess the credibility of prior claims. Rarely is a single follow up investigation sufficient. Research may go on for years challenging, debating, and refining claims. Sometimes it is difficult or impossible to obtain additional evidence; A decision must be made with what is already available.

The "Systematizing Confidence in Open Research and Evidence" (SCORE) program has an aspirational objective to develop and validate methods to assess the credibility of research claims at scale with much greater speed and much lower cost than is possible at present. Imagine it takes a year to achieve $95 \%$ accuracy in assessing the credibility of a claim by conducting replication and generalizability studies, a month to achieve $85 \%$ accuracy by conducting reproduction and robustness tests of the same claim, and a few hours to achieve $80 \%$ accuracy by consulting a group of experts to review the readily available evidence. Could we create automated methods to achieve similar accuracy as experts in a few minutes or a few seconds? If that were possible, readers, researchers, reviewers, funders, and policymakers could use the rapid assessments to direct their attention for more laborious assessment and improve allocation of resources to examine claims that are important but relatively uncertain.

There is accumulating evidence that such a service is needed and possible to achieve. In the social and behavioral sciences, replication efforts have indicated that the literature is not as replicable as might be expected (Camerer et al., 2016, 2018; Cova et al., 2018; Ebersole et al., 2016, 2020; Klein et al., 2014, 2018; Open Science Collaboration, 2015). For example, Nosek and colleagues (2021) aggregated 307 replication attempts of published findings in psychology and observed that $64 \%$ reported statistically significant evidence in the same direction as the original studies, with effect sizes $68 \%$ as large as the original studies. Investigations of robustness and reproducibility of claims suggest that some published evidence is highly contingent on specific analytic decisions, or even irreproducible (Botvinik-Nezer et al., 2020; Silberzahn et al., 2018; Simonsohn et al., 2020). These investigations indicate that the credibility of published claims is more uncertain than expected.

Multiple studies indicate that people can anticipate which findings are likely to replicate after reading the original paper or even just reviewing a subset of information about the finding and supporting evidence (Camerer et al., 2016, 2018; Dreber et al., 2015; Forsell et al., 2019; Wintle 
et al., 2021). Human judgments were correlated with successful replication using prediction markets $(r=0.52)$, surveys $(r=0.48)$, and structured elicitations $(r=0.75$; see Nosek et al., 2021 for a review). This suggests that relatively accurate credibility assessments are achievable with an order (or orders) of magnitude lower resource investment than conducting replication or reproduction studies.

Finally, three studies provide initial evidence that machine learning methods may provide a scalable solution that could match, or perhaps even exceed, the capabilities of human judgment (Altmejd et al., 2019; Pawel \& Held, 2020; Y. Yang et al., 2020). Each machine learning investigation used a distinct approach drawing on narrative text of the original paper, information about original designs and replication sample sizes, or other contextual information about the original finding. These promising findings provide a basis for SCORE's primary goal to investigate scalable methods of assessing credibility of claims in the social-behavioral sciences.

SCORE began in February 2019 and the main activities will conclude in May 2022. This paper introduces the program structure, activities, and expected outcomes of the program, including data and artifacts that will be made available to the research community for further investigation.

\section{Program Scope and Structure}

SCORE is a large-scale collaboration involving eight primary research teams and more than a thousand contributing researchers. The teams are organized into three technical areas (TAs) TA1, TA2, and TA3 - and a Testing and Evaluation (T\&E) group that evaluates the TAs and program effectiveness. The primary research teams have clearly specified roles, distinct areas of expertise, and shared objectives organized around a common set of articles constituting the shared Common Task Framework (CTF). The research teams work with the shared CTF dataset in a coordinated way to advance the SCORE program's goals (see Figure 1).

The CTF consists of approximately 30,000 articles from 2009-2018, representing 62 journals from the following disciplines: Criminology, Economics and Finance, Education, Health, Management, Marketing and Organizational Behavior, Political Science, Psychology, Public Administration, and Sociology (see Supplement Table 1). From the CTF, a stratified random sample of 3,000 papers was selected for additional investigation and enhancement, called the annotation set. Stratification ensured that the annotation set had approximately equal representation of papers per year per journal. From the annotation set, a stratified random sample of 600 papers was then sampled for additional investigation such as conducting reproduction or replication studies, called the evidence set. Stratification again ensured approximately equal sampling of papers per year per journal. This sampling was done without regard to the feasibility of any particular empirical attempt, with the understanding that not all claims will receive a completed empirical study result. This design is intended to be adaptive to the resource-intensiveness of different activities for assessing credibility while also maximizing the generalizability of the findings to the social-behavioral sciences. 
The purpose of the team structure and shared set of papers is to investigate the credibility of claims from the social-behavioral sciences and test methods for efficiently assessing that credibility. To do this, the project is organized in modular stages with specific responsibilities for each team.

TA1, the Center for Open Science (COS), is responsible for enhancing the CTF database and extracting claims for the annotation set to be evaluated by the other teams. In the completed half of the program, this meant extracting 3,000 individual claims, one from each paper. TA2 teams from KeyW/Jacobs Corporation and University of Melbourne used human evaluators to provide confidence scores predicting the reproducibility or replicability of the 3,000 research claims in the annotation set. These teams competed with each other to provide the most accurate scores. Three TA3 teams from Pennsylvania State University (PSU), TwoSix Labs, LLC, and University of Southern California (USC) used machine learning methods to develop algorithms that assign confidence scores just like the human evaluators.

Figure 1. Relationships between research teams comprising the three technical areas (TAs) of the SCORE program.

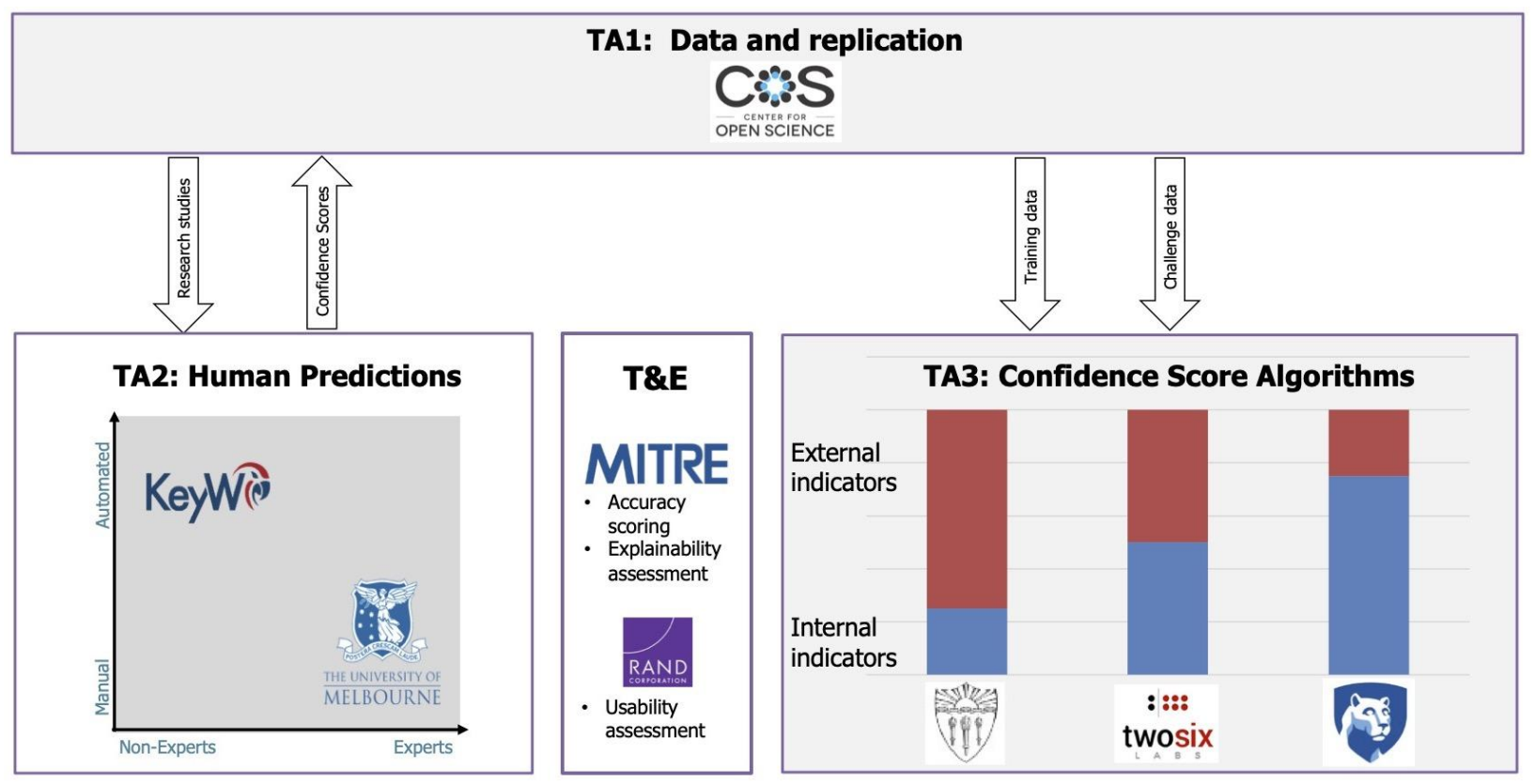

While TA2 and TA3 teams generated scores for these 3,000 claims, TA1 privately created a stratified random sample of 600 of those papers to create the evidence set. Some claims from the evidence set were subjected to reproduction and replication studies. TA2 and TA3 teams were left unaware of which claims were selected for the evidence set to avoid any complications of altering strategy to focus on specific claims. The reproduction and replication outcomes provide a ground truth benchmark for evaluating accuracy of the confidence scores generated by humans, a process managed by the Testing and Evaluation (T\&E) teams. Algorithms are evaluated primarily on their ability to predict the human credibility assessments across all 3,000 claims, and assessed for explainability of the generated confidence scores. Which claims were 
selected for replication or reproduction studies, and the outcomes of those studies were held back from TA2 and TA3 teams until their credibility scores are committed and completed.

Entering the second half of the program, the breadth and depth of the project is expanding with TA1 sampling additional claims from the CTF, extracting a single claim per paper for another 900 papers, and systematically extracting a complete "bushel" of claims from 200 of the initial 600 papers in the evidence set. The complete set of bushel claims is meant to represent all of the claims that could have been selected from the paper in the first half of the program, rather than simply the one claim that was selected. The Melbourne TA2 team is expanding the task of the human evaluators to evaluate all of the bushel claims and to assess those papers on multiple indicators of credibility. TA3 teams are extending their strategies for improving algorithm performance. And, finally, TA1 is expanding the scope of assessing reproduction, robustness, and replicability for the evidence set of 600 papers.

\section{What Makes SCORE Unique}

SCORE is inclusive of a substantial portion of the social-behavioral sciences to facilitate generalizability and investigation of heterogeneity in credibility and replicability across subdisciplines and methodologies. Also, with a standard identification process of discrete claims across papers, the SCORE program facilitates broad inclusion of outcome types, comparison of those outcomes across papers, and a variety of verification attempts including reproduction, robustness, and replication tests.

Another virtue of the SCORE program is that it includes many distinct efforts on the same large dataset, facilitating the opportunity for comparative analysis. For example, the most enriched papers from the evidence set will have structured claim extraction from the paper, metadata about the paper from external databases (e.g., citation rates, presence of open data), human credibility scores from multiple sources, machine credibility scores from multiple sources, and evidence on reproducibility, robustness, and replicability of one or multiple claims.

Finally, at the conclusion of the program, SCORE data will be accessible to others for research. Additional users of SCORE data may themselves enhance the dataset and other artifacts creating a generative, virtuous cycle of data enrichment fostering new investigations that provide further enrichment.

\section{Defining and Extracting Scientific Claims}

The TA1 team annotates the papers randomly sampled into the annotation set. In the completed part of the project, this meant identifying a single relevant claim from each paper, by tagging related information in an article. In SCORE terminology, this claim represents a specific, concrete finding that is supported by a statistically significant test result, or at least by evidence that would be amenable to a statistical hypothesis test even if the authors did not adopt significance testing. This is not the only way to identify a claim, but this working definition provides clarity between teams, sufficient flexibility to cover a wide range of research applications, and is sufficient constraint to define criteria for evaluating confidence and 
assessing replicability and reproducibility. Table 1 shows a glossary of SCORE's working definitions.

Table 1: A glossary of key terms as they are used for the SCORE program

\begin{tabular}{|c|c|}
\hline Paper & $\begin{array}{l}\text { A single academic article that makes quantitative claims based on specific } \\
\text { social scientific data. SCORE does not address papers that are exclusively } \\
\text { based on qualitative research, simulations, theory, or commentary. }\end{array}$ \\
\hline $\begin{array}{l}\text { Common Task } \\
\text { Framework (CTF) }\end{array}$ & $\begin{array}{l}\text { The set of approximately } 30,000 \text { papers that constitutes the sampling frame for } \\
\text { SCORE. It includes papers from } 62 \text { social science journals published between } \\
2009 \text { and } 2018 \text {. }\end{array}$ \\
\hline Annotation Set & $\begin{array}{l}\text { A stratified random sample from the CTF of approximately } 3,000 \text { papers that } \\
\text { are annotated to identify at least one claim trace per paper. }\end{array}$ \\
\hline Evidence Set & $\begin{array}{l}\text { A stratified random sample of approximately } 600 \text { papers from the annotation } \\
\text { set. These papers could be selected for an empirical attempt to find further } \\
\text { evidence for or against a claim. }\end{array}$ \\
\hline Claim & $\begin{array}{l}\text { A specific assertion reported as a finding in a paper. Most papers make more } \\
\text { than one claim, and claims in a paper can be related or independent of one } \\
\text { another. }\end{array}$ \\
\hline Claim Trace & $\begin{array}{l}\text { A claim in a paper is identified by annotating and labeling short excerpts from } \\
\text { the main text or tables/graphs from the paper. Together these annotations let a } \\
\text { reader 'trace' from a general statement in the abstract to a more specific claim } \\
\text { to the quantitative information such as a specific inferential test or estimate that } \\
\text { is given as evidence for that claim. }\end{array}$ \\
\hline Confidence Score & $\begin{array}{l}\text { A prediction about the replicability of a claim, expressed as a numerical value } \\
\text { on a scale from "not confident" to "very confident." Confidence scores are about } \\
\text { a single claim which may or may not generalize to confidence in other claims } \\
\text { from the same paper. }\end{array}$ \\
\hline Inferential Test & $\begin{array}{l}\text { A statistical calculation that supports an inference about a single effect and } \\
\text { provides information about both the spread and central tendency of that effect. } \\
\text { When testing statistical significance, a single inferential test is associated with a } \\
\text { single p value. Additionally, with regression modeling, inferential tests may be } \\
\text { associated with a single parameter, or with an entire model if model comparison } \\
\text { tests are conducted. }\end{array}$ \\
\hline Bushel Claim & $\begin{array}{l}\text { A set of claim traces from a single paper representing as many of the } \\
\text { independent claim traces that the authors present as possible. Each claim trace } \\
\text { must be linked to a finding reported in the abstract, and must be supported by } \\
\text { quantitative evidence presented in the main text. }\end{array}$ \\
\hline Empirical Study & $\begin{array}{l}\text { A single empirical attempt conducted by a research team to provide additional } \\
\text { evidence about a claim. These attempts can include conducting a replication, } \\
\text { reproduction, or other empirical activity that speaks to the credibility of that } \\
\text { claim. }\end{array}$ \\
\hline Replication & $\begin{array}{l}\text { Testing the reliability of a prior finding with new data expected to be theoretically } \\
\text { equivalent by comparing the outcome of an inferential test as reported in a } \\
\text { paper with the equivalent inferential test as calculated in the new dataset. }\end{array}$ \\
\hline
\end{tabular}




\begin{tabular}{|l|l|}
\hline Reproduction & $\begin{array}{l}\text { Testing the reliability of a prior finding with the same data and same analysis } \\
\text { strategy by comparing the outcome of an inferential test as reported in a paper } \\
\text { with a re-calculation of that inferential test from the original data. }\end{array}$ \\
\hline Robustness & $\begin{array}{l}\text { Testing the reliability of a prior finding with the same data and different analysis } \\
\text { strategy by conducting alternative tests on the original data. }\end{array}$ \\
\hline Generalizability & $\begin{array}{l}\text { Testing the reliability of a prior finding in a new dataset in a way that differs from } \\
\text { the original study but is expected to produce similar results. }\end{array}$ \\
\hline
\end{tabular}

Table 2: A single claim trace of a paper is composed of four levels.

\begin{tabular}{|l|l|}
\hline Claim 1 & The title of the paper. \\
\hline Claim 2 & A statement from the paper's abstract that reflects an empirical research finding. \\
\hline Claim 3 & $\begin{array}{l}\text { A hypothesis, prediction, or finding statement presented somewhere in the main } \\
\text { text of the paper, relating to the finding reported in Claim 2. }\end{array}$ \\
\hline Claim 4 & $\begin{array}{l}\text { A result supported by specific statistical information in the article that supports } \\
\text { Claim 3, alongside the authors' interpretation of that information. }\end{array}$ \\
\hline
\end{tabular}

Figure 2. Model of a bushel claim set for a single paper. Each line represents a distinct bushel claim trace. Two examples of single-trace claims that could have been extracted are in blue and red.

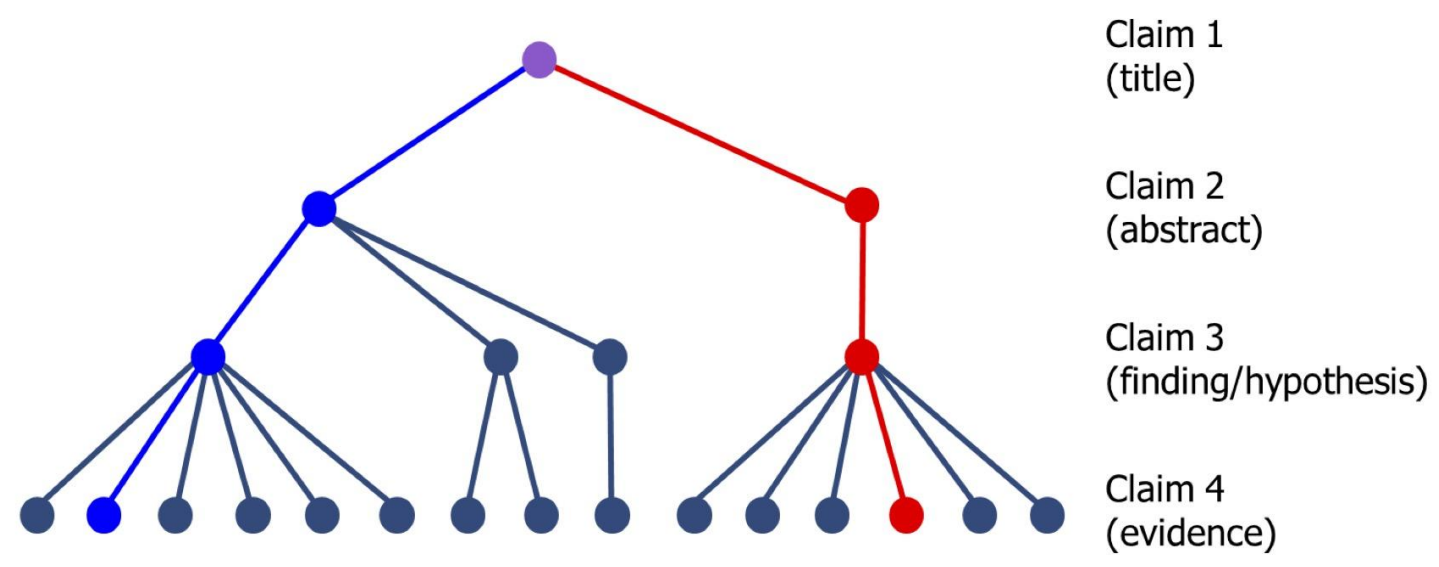

The output of the annotation process is a "claim trace" that maps a finding reported in the abstract to a specific hypothesis or finding from the main text, to a particular set of quantitative evidence that supports the reported finding. When only one claim trace is identified, the process does not guarantee that the claim trace selected necessarily includes the paper's "most important" or "most central" claim. Pretesting revealed that this kind of decision is neither objective nor obvious for many papers. Instead, as a proxy for a lower bound on importance, a claim must be directly related to a statement made in the paper's abstract. This criterion avoids 
selecting tangential findings unrelated to the summarized purpose of the paper. The claim trace indicates a series of levels leading down to the specific focal result as described in Table 2.

Selecting a single finding creates a tractable and comparable way for independent teams to work with a paper, and it has clear limitations for interpreting the results. Papers often include more than one finding in the abstract, and research findings are often supported by multiple pieces of evidence. In the current phase of work, we have expanded claim extraction for some papers in the evidence set by adding a second bushel approach that relaxes these requirements. In the bushel approach, we identify as many unique claims as possible by tracing from a finding in the abstract to statistical evidence in the paper. In addition, we relax the definitions of evidence to allow tagging of multiple inferential tests and other types of quantitative evidence. Figure 2 illustrates a bushel of claims from a paper and two single-trace claims that could be extracted.

\section{Expert Assessment}

The second major technical area (TA2) elicits predictions, called confidence scores, from human readers about replicability of extracted claims. TA2 included two independent teams, repliCATS and Replication Markets, to examine the viability and accuracy of distinct forecasting strategies.

\section{repliCATS - Structured elicitations}

The repliCATS (Collaborative Assessments for Trustworthy Science) project uses a structured elicitation process--the IDEA protocol--to complete group evaluations of research claims. IDEA stands for: Investigate, Discuss, Estimate, and Aggregate (Figure 3). IDEA is a modified form of the Delphi protocol, with the major differences being that IDEA encourages interaction between participants and does not require behavioural consensus. Interaction between participants occurs in online comments, sometimes supplemented with face-to-face discussions. In the first half of the program, repliCATS assessments focused on predicting the likely replicability of research claims. In the remainder of the program, the scope of assessments is expanding to also include other judgements of research credibility, e.g., transparency, robustness, validity and generalizability.

In repliCATS, experts work in small groups, using a custom built cloud-based elicitation platform (Fraser et al., 2021; Pearson et al., 2021). Individual experts first make their own private estimate of whether or not the identified claim will replicate and document their reasons (Investigate). After lodging their initial estimates, individuals receive feedback about their group members' judgements and reasoning, and can compare their own judgements. Groups are encouraged to interrogate differences of opinion and share relevant information (Discuss). Following discussion, each individual provides a second private assessment (Estimate). Mathematical aggregation of individual estimates removes the need for group members to reach a consensus (Aggregate). 
Figure 3. Overview of the IDEA protocol, as adopted in the repliCATS project

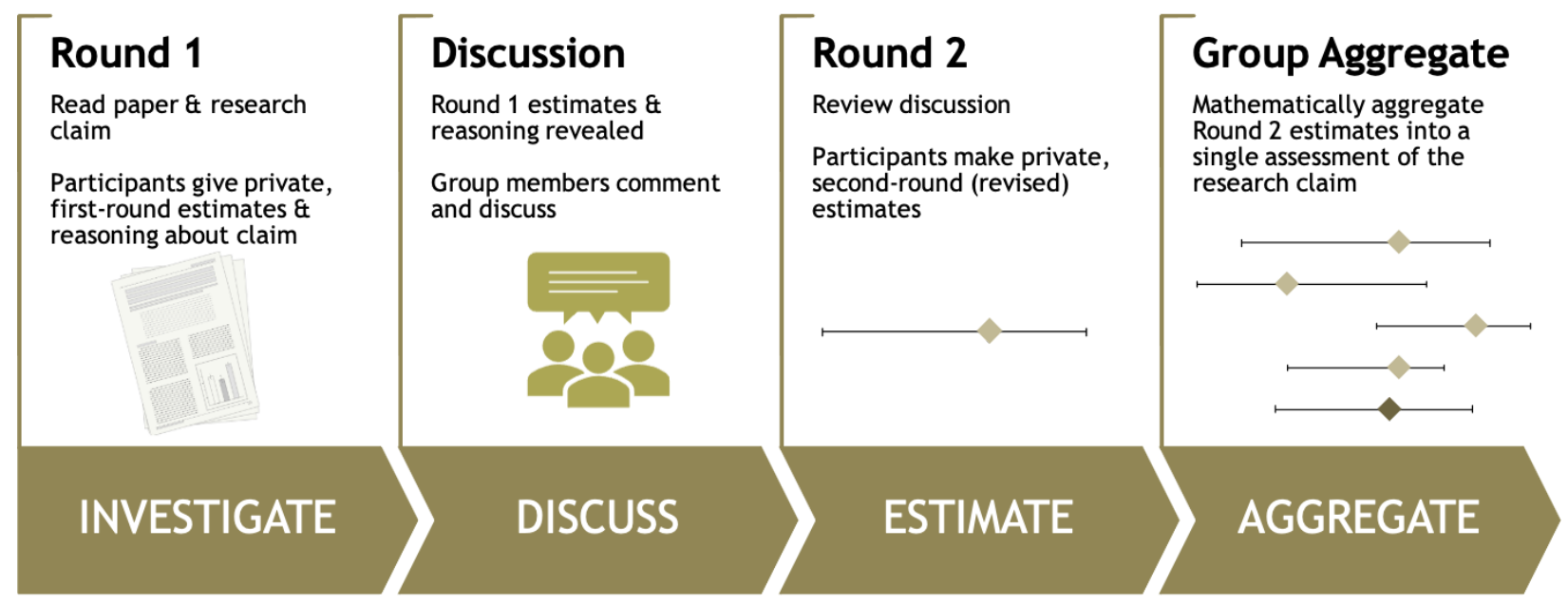

The repliCATS project has several preregistered aggregation models (https://osf.io/m6gdp/). Described in detail by Hanea and colleagues (2021), the aggregation models fall into three categories: (1) linear combinations of best estimates, transformed best estimates (Satopää et al., 2014) and distributions (Cooke et al., 2021); (2) Bayesian approaches, one of which incorporates characteristics of a claim directly from the paper, such as sample size and effect size; and (3) weighted linear combinations of best estimates, mainly by potential proxies for good forecasting performance, such as demonstrated breadth of reasoning, engagement in the task, openness to changing opinion and informativeness of judgments (Mellers, Stone, Atanasov, et al., 2015; Mellers, Stone, Murray, et al., 2015). The third category of models is the largest.

The structured elicitation protocol and deliberate inclusion of text responses on the repliCATS platform will create a rich qualitative database, with experts documenting the reasoning behind their predictions and judgements. Justifications commonly focus on design issues, sample size, and papers' overall clarity and logical structure.

\section{Replication markets}

Replication Markets extends work showing that markets of domain experts can accurately estimate the replicability of findings in the social and behavioral sciences (Camerer et al., 2016, 2018; Dreber et al., 2015; Ebersole et al., 2020; Forsell et al., 2018; Klein et al., 2018; Gordon et al., 2021). In many contexts (K.-Y. Chen et al., 2003; Forsythe et al., 1992, 1999), markets appear to provide better estimates than any individual, especially in complex combinatorial prediction markets (Y. Chen \& Pennock, 2010) where individuals make systematic errors (Wang et al., 2011).

However, where those markets forecast replicability of 18-40 similar claims at a time, all of which would be tested, SCORE forecasted 3,000 highly diverse claims in about a year, with only a small fraction to be resolved by experiment. We elicited forecasts in 10 monthly rounds of 
300 claims, using a decision market mechanism to preserve proper incentives given the low resolution rate (Figure 4).

Each round of claims had one week for surveys followed by two weeks of markets. In markets, forecasters traded 'Yes' and 'No' shares on binary replication questions. 'Yes' shares pay 1 point if the replication yields a statistically significant finding in the direction of the original claim. Otherwise 'No' shares pay 1 point. Surveys directly solicited chances on replications. A total prize pool was split $(\sim 2 / 3)$ to the prediction markets, and $(\sim 1 / 3)$ to the survey. Market prizes are paid when replication outcomes become available. Survey prizes were paid each round after the markets closed, using surrogate scores (Liu et al., 2020).

Surrogate scoring scores a forecast based solely on reported forecasts across claims made by other forecasters. It exploits the unknown statistical correlation of forecasts. Under certain conditions and with enough claims and forecasts, a forecaster's expected surrogate score reflects their true (unknown) forecast accuracy, and surrogate scoring incentivizes truthful forecasting. For instance, in expectation a forecaster's surrogate score equals their true (unknown) Brier score. Thus, surrogate scoring allows us to provide immediate, potentially noisy, feedback on forecast accuracy before replication outcomes become available. Once the outcomes are available, we will evaluate forecasting performance according to reregistered tests (Pfeiffer et al., 2020). Replication markets and surrogate scoring were also used to forecast the overall SCORE replication rate by field and publication year (Gordon et al., 2020).

Figure 4. Overview of the Replication Markets workflow.

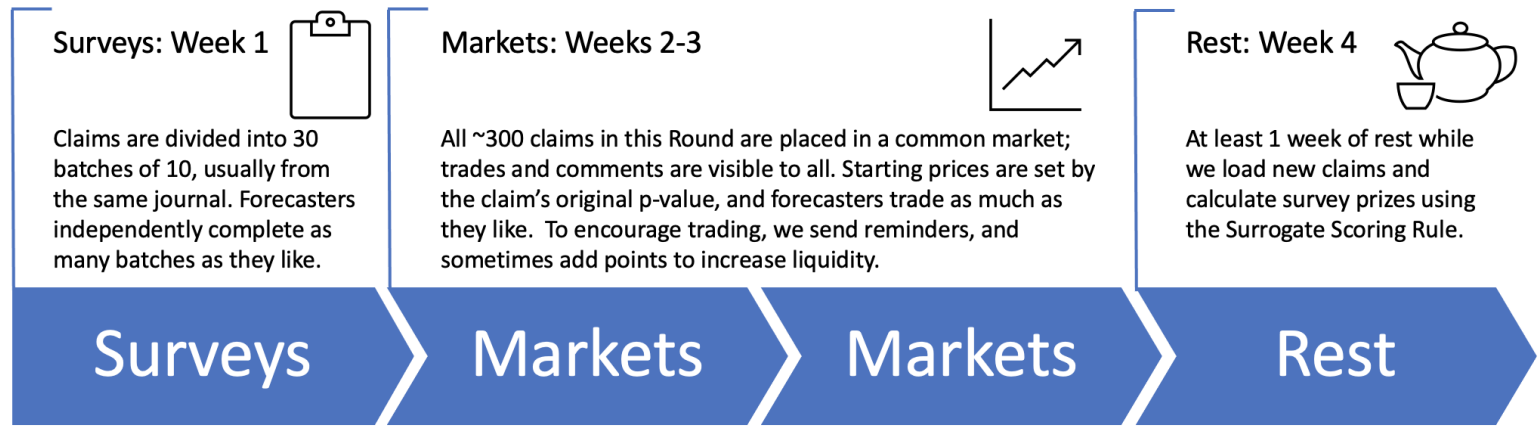

A replication markets "Round" has 300 claims, three weeks of forecasting, and a rest of usually 1 week.

\section{Machine Assessment}

The third technical area (TA3) uses the same dataset of extracted claims to generate confidence scores using machine learning and other algorithmic approaches. The three teams -- PSU, TwoSix, USC -- use different approaches for generating confidence scores.

\section{PSU}

Researchers at Pennsylvania State University, in collaboration with others at Texas A\&M University, Old Dominion University, and Rutgers University use synthetic prediction markets for scoring the replicability of claims. As with the human Replication Market team, a research claim 
is treated as a binary option in which the price of the option of a claim at market close can be interpreted as an indicator of confidence in its replicability. Within this framework, artificial agents, or trader-bots, are endowed with initial cash and may choose to purchase options of a given claim. Bots are trained using an evolutionary algorithm and data from existing replication studies (e.g., Open Science Collaboration, 2015) and expert assessments provided by TA2. Trade logs provide explanatory power.

In the prototype, asset prices for claims were determined by a logarithmic market scoring rule. Artificial agents were endowed with purchase logic defined using a sigmoid transformation of a convex semi-algebraic set defined in feature space (Nakshatri et al., 2021). The team's feature extraction and representation (FEXRep) framework extracted 42 bibliometric, bibliographic, statistical and semantic features from each paper (Lanka et al., 2021; Modukuri et al., 2021; Wu et al., 2021, 2020). The team is expanding feature extraction capabilities to support evaluation of bushel claims, incorporating more claim-level features and information about the relationships amongst multiple claims in a paper. The next phase will include hybrid market experiments wherein human traders will participate alongside artificial agents.

PSU's synthetic market approach is inspired by the success of prediction markets in estimating reproducibility of scientific research and motivated by their shortcomings. Prediction markets require the coordinated, sustained effort of collections of human experts limiting their feasibility to scale. They rely on the availability of well-defined and verifiable outcomes which are determined after market close. Synthetic prediction markets can be deployed rapidly and at scale. They can be updated continuously as new information becomes available with periodic, offline human input. Agents can have comprehensive access to prior scholarship far beyond the capacities of an individual researcher. The group has dedicated effort to developing a comparable baseline ("Red Team") led by Texas A\&M University and leveraging state of the art approaches for interpretable representation learning developed within DARPA's XAI program (Du et al., 2021, 2018; F. Yang et al., 2018).

\section{TwoSix}

The A+ system developed by Two Six Technologies is a method for understanding replicability given only a journal article, illustrated in Figure 5. The A+ system contains three major computational components: semantic parsing, feature extraction, and replication prediction.

Semantic parsing. After extracting text from the PDF using Automator, A+ represents the overall semantic context of each section. This is similar to prior annotation work (K.-Y. Chen et al., 2003; Dasigi et al., 2017; Huber \& Carenini, 2019). Modifying the annotation scheme better matched the problem of information extraction for replication prediction. The discourse class for each sentence is inferred and then averaging of the outputs to obtain the final class.

Information extraction. The unstructured prose of scientific documents includes key features for assessing replicability, such as sample sizes, populations, conditions, experimental variables, methods, materials, exclusion criteria, and participant compensation. Much of this 
information is available as concise spans of text in the document: "twenty-four" may be a sample size; "undergraduates" may be a population description; "reaction time" may be a dependent variable. Consequently, we are not interested in extracting and classifying relations at this phase of analyses; rather, the information extractor is optimized to classify individual spans within the text with context-sensitive labels (e.g., sample count and characteristics, experimental variables, methods), to create a dataset of 620 annotated examples. The model also processes the resulting classified spans to opportunistically extract domain-specific numerical and Boolean features. For example, the sample and exclusion counts are expected to be integers, so it attempts to coerce "one hundred and ninety - seven" and "Eight" to integers. Similarly, the model uses a lexicon-based approach to populate Boolean features indicating whether participants' genders, age, race, religion, and community are specified, what the recruitment pool is (e.g., AMT, universities), and how they are compensated (e.g., course credit, monetary). Because statistical tests are more structured than these features, specific Python regular expressions are applied to identify 25 different statistical tests and values including $p, R$, $\mathrm{R}^{2}$, d, F-tests, T-tests, mean, median, standard deviation, confidence intervals, odds ratios, and non-significance.

Figure 5. Illustration of the A+ pipeline. Manuscripts are first tagged based on semantic analysis and then specific aspects of information are extracted from tagged portions of the manuscript's body. The article's structure and extracted information serve as features for a confidence score prediction model.

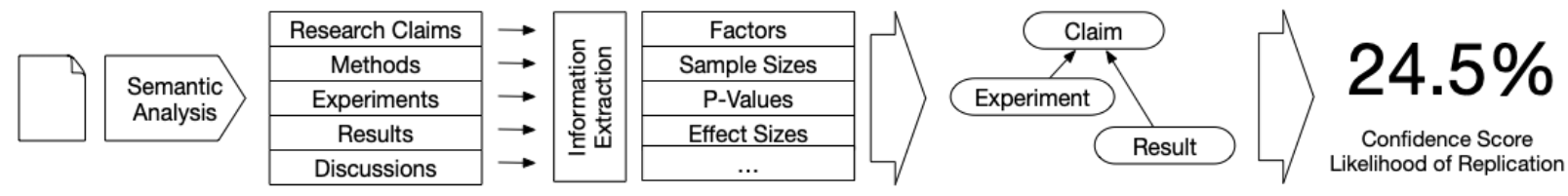

After extracting individual spans and subgraphs, it is assembled into a global graph called the argument structure. As implied by its name, the argument structure expresses the premises, evidence, and observations in a scientific article, ultimately in support of its conclusions. The system generates the argument structure by iterating over the sequence of text segments and semantic tags to create a structured set of nodes representing the article. Upon encountering a transition in semantic tags, such as a new Methodology section after a Discussion section, the system instantiates a new Study node and adds the appropriate features.

Replication prediction. The graph-based layout of the argument structure allows the system to assess independent replicability concerns in a context-sensitive, explainable fashion. For example, a sample size of 24 for a study node may impact the judgment of that study's replicability, but it does not necessarily impact the replicability judgment of another study in the paper. Each node in the directed argument structure graph is connected directly or indirectly to the node representing the scientific article. The argument structure is a fully-connected graph that supports graph and pattern matching, confidence propagation, and feature extraction to judge and explain replicability. 


\section{University of Southern California}

The MACROSCORE system developed by the University of Southern California is a knowledge fusion system that captures a holistic view of the factors important for reproducible and replicable research. The approach mimics the complex judgments that human reviewers make when assessing research. The first pipeline relies on "micro"-features: those that are based on information extracted from papers pertaining to the parameters of the study (e.g., study design, sample population, preregistration, open data). Potential detractors to scientific validity, such as conflicts of interest, are also extracted. MACROSCORE uses an adaptation of SciBERT, a pre-trained language model created using millions of scientific papers, to identify entities such as experimental parameters, open science indicators, and claim information.

The second pipeline in MACROSCORE is the "macro"-feature pipeline that captures the broader scientific context of a paper. Determining the impact and contributions of a scientific work is a difficult and subjective task. MACROSCORE collects the citations and references within a particular scientific discipline, forming a network connecting the scientific articles and their authors. Metrics of network structure, including in-degree (incoming citations to the work), out-degree (references to other works), authority score (citations by important works), and hub score (citing important work) assess the scientific work.

The heart of the MACROSCORE system is a knowledge graph that represents the data from micro and macro pipelines. The knowledge graph represents the core concepts of the scientific discipline: scholarly works, scientific claims, scholars, organizations, and publication venues. MACROSCORE uses an ontology from the popular knowledge graph Wikidata to include each scientific article, the journal where it was published, its authors and editors, and the affiliations of each, and all citations and references. MACROSCORE has extended the ontology on Wikidata to incorporate claim information and derived features from four high-level classes: validity of inference, study design, reporting and transparency, and scientific network. Together, these features create a comprehensive profile of the paper and its connection to other works.

The final component of the MACROSCORE system is a suite of predictive algorithms that operate on the features from each pipeline and the knowledge graph. MACROSCORE uses a probabilistic graphical model using the probabilistic soft logic (PSL) framework. This model includes dependencies between different features defined in the knowledge graph specified as logical rules, such as "small sample sizes and small effect sizes indicate poor replicability." Using training data, the PSL framework learned the importance of each rule and its associated features. For a given judgment made by the MACROSCORE system, the PSL model will provide a set of explanatory statements, and an analysis of the top features contributing to the assessment.

\section{Empirical evidence for credibility assessment}

Independent empirical assessments provide the basis for evaluating the confidence scores generated by humans and algorithms to predict credibility of claims. Table 4 presents approaches to empirical assessment of credibility roughly ordered from the bottom being the least effortful but providing the least information about a phenomenon to the top being the most 
effortful and providing the most information. In general, lower categories in Table 5 correspond with assessments of the original design and original data for a narrow test of whether the original report found what it reported to have found, and higher categories correspond with more laborious assessments involving obtaining new designs and data for a broader test of whether the original claim is supported by new evidence. These are not the only ways to assess credibility. For example, a finding could be reproducible, robust, replicable, generalizable, and invalid if the interpretation is incorrect. Nevertheless, these assessments are tractable and verifiable indicators that are related to other aspects of credibility.

As TA1, COS bears responsibility for coordinating a large network of social-behavioral researchers to contribute empirical evidence assessing the credibility of claims. The team draws on the stratified random sample of 600 claims comprising the evidence set and matches their topics and methodologies to researchers with appropriate resources and expertise to conduct an empirical assessment. The focus of the first half of the SCORE program was on conducting replication and reproduction studies. The remainder of the program expands the scope of empirical evidence to include all of the forms presented in Table 3.

Table 3. Forms of empirical credibility assessment

\begin{tabular}{|c|c|}
\hline Generalizable & $\begin{array}{c}\text { Original claim supported across diverse samples, } \\
\text { treatments, outcomes, and settings }\end{array}$ \\
\hline Replicable & Original claim supported with independent evidence \\
\hline Robust & $\begin{array}{c}\text { Original claim supported with diverse treatments of } \\
\text { original data }\end{array}$ \\
\hline Outcome Reproducible & Original claim supported with original analysis of \\
original data
\end{tabular}

A reproduction refers to applying the original analysis strategy to the original data to test whether the same result recurs. A reproduction could fail due to process reproducibility because, for example, the original data are not available, making it impossible to conduct the analysis again. This does not disconfirm the finding, but it is a credibility risk in that the finding cannot be confirmed or disconfirmed. A reproduction could also fail due to outcome reproducibility because, for example, applying the analysis described in the original paper does not produce the finding associated with it. This can occur because of errors in reporting, ambiguity in description of analyses, or factors in the data analysis pipeline. 
A replication refers to testing the original claim with different data. That data could be pre-existing, such as re-testing the relationship between variables in a subsequent wave of a panel study, or could be newly generated with a study design to test the same research question. Whether based on existing or new data, the determination of whether a new test is a replication of a prior claim is a theoretical commitment that the inevitable differences between the original and replication study are irrelevant for testing the original claim (Nosek \& Errington, 2020).

To provide evidence that is both appropriate to testing individual claims and standard enough to evaluate SCORE teams' prediction methods across disciplines, we designed a process that balances specific requirements that all projects must adhere to with ongoing evaluation and feedback by subject area experts. For example, all replications are prepared using a standard template that is reviewed by 2-3 independent researchers, and the resolution of design changes suggested by reviewers is managed by an editor. Authors of the original finding are invited to review or submit a commentary on the design. The review process is intended to improve the quality of the replication designs so that they are effective, good-faith tests of the original claim. The template and review process also provide an occasion to explicitly document differences between original and replication studies and assessments of any heterogeneity in beliefs about whether they are consequential for the replication design. Following approval, the design and analysis plan is preregistered on the Open Science Framework (OSF). Research teams conduct their studies and then report outcomes following a standard protocol and provide all research materials, data, and code so that the replication studies will be themselves reproducible and, eventually, accessible to others to the extent ethically possible. The reproduction workflow has a similar emphasis on documentation and transparency with a lighter review process emphasizing adherence to the standardized protocol for reproducing original findings.

As singular attempts to reproduce or replicate original claims, these empirical efforts will not provide definitive evidence about their credibility (Open Science Collaboration, 2015) -- they add to the body of evidence about that claim which includes the original paper and any other evidence in the literature. However, prior evidence that both humans and algorithms can predict the outcomes of these reproductions and replications provides a basis for treating them as ground truth for the purposes of the program. More importantly, the generated dataset of original and novel statistical evidence, reproduction and replication outcomes, along with the expanded set of empirical credibility indicators from internal consistency (e.g., statcheck), robustness (e.g., multiverse or many-analyst investigations), or generalizability tests will provide a rich network of evidence to investigate convergence and heterogeneity of these credibility indicators.

\section{Evaluating Expert and Machine Success}

There is no definitive criterion for deciding whether a finding is successfully replicated or reproduced (Nosek et al., 2021), but pragmatic, defensible, and widely applicable benchmarks are needed to evaluate the outcomes of the SCORE program. The role of the MITRE Test \& Evaluation (T\&E) team in SCORE is to evaluate the relative match between predicted and actual confidence in each claim using the outcomes from the TA1 empirical results and the 
human-generated confidence scores from TA2. T\&E focuses on evaluating the accuracy of human-generated confidence scores relative to replication outcomes and the accuracy of algorithm-generated confidence scores relative to the most accurate human-generated scores.

T\&E's evaluation of TA2's aggregated human confidence scores against TA1 binary replication outcomes is operationalized through various metrics. Primary emphasis is given to a modified area under the curve (AUC) (aka "signal detection") metric, which can be interpreted as the "meta-probability" of TA2 assigning a higher confidence score to a claim that does indeed replicate than to a claim that fails to replicate -- for any given randomly sampled pair of such cases (Pepe, 2003; Steyvers et al., 2014). Supplementary metrics include proper scoring rules (Brier, 1950), measures of calibration (Arkes et al., 1995), and measures of association between TA2 confidence scores and replication $p$-values (where smaller $p$-values indicate higher levels of replication study support for the original study claim).

To evaluate algorithm accuracy in predicting human confidence scores, the root mean squared error (RMSE) is used as one of two primary outcome metrics. Additionally, Kendall's tau-b, a nonparametric measure of monotonic association (Gibbons \& Fielden, 1993) is used to assess accuracy in discriminating among claims with greater or lesser amounts of replication support. Finally, we use measures of calibration as a supplementary metric (e.g., regression of TA2 scores on TA3 scores, where intercept and slope deviating from 0 and 1, respectively, would be evidence of miscalibration).

RAND researchers will pilot the use of TA3 tools to assess their applicability with users in the policy community. While few studies have an explicit emphasis on the reproducibility of scientific claims, matters of generalization and reliability weigh heavily on the development and assessment of policy interventions. Two applications of particular interest include the ability to characterize findings from large bodies of literature that form the initial basis of information from which further studies are drawn, and in the role of adjudicating load-bearing claims that may be sources of contention among policy making stakeholders.

We do not have outcomes to report for SCORE yet. However, interim analysis of performance of expert and machine teams indicates viability of the approach sufficient to obtain approval from DARPA to enter the second phase of the program. A full report of outcomes will follow conclusion of the program and consolidation of all available credibility evidence.

\section{Potential Outcomes, Findings, and Artifacts}

The primary research objective for SCORE is to create accurate, scalable, automated algorithms to signal confidence in research claims. If the program outcomes meet this aspirational objective, there are a variety of potential use cases. Researchers might use scores to identify potential weaknesses in their claims and provide more detail or support. Journal editors and conference organizers might use the scores to prioritize selection of reviewers with expertise in areas that the algorithms flagged as low confidence. Funders and researchers designing proposals might use the scores to identify potentially important findings that have not yet achieved high confidence. The scores could guide policymakers' information search and 
allocation of effort to obtain additional evidence or expert judgment when the algorithms flag uncertainty.

Even the most optimistic assessments of the potential of such scores would not defer reasoning, decision-making, judgment, and action to machines. Uncritical use of algorithms can perpetuate biases in how we evaluate claims, or reflect inappropriate generalizations about what signals indicate that a paper is credible (Buolamwini \& Gebru, 2018; Caliskan et al., 2017; Larson et al., 2016). Effective automated technologies can be a tool to complement these human and social processes in the assessment, prioritization, and application of research. They can also provide researchers with tools for rapid and iterative assessments of credibility. At scale, as an iterative feedback mechanism, they may help foster culture and behavioral changes that increase the overall credibility of research.

SCORE represents a unique opportunity to explore a challenge that is paramount to modern Al--How can we combine the best of both human and machine reasoning? Explainability of results in machine learning is always challenging, but it is made more so by the nuance inherent in human writing and scientific expression beyond the more tractable reporting of statistical information. With multiple algorithm strategies using enriched extracted information from papers and human judgment and replication outcomes as validation measures, SCORE may facilitate significant progress on this problem.

Even if the primary objective is not realized, SCORE will advance a variety of research questions about the credibility and assessment of scholarly research, and generate research artifacts that can support dozens or hundreds of investigations. These artifacts will include:

1. Annotation Set: A stratified random sample of 3,000 papers with a claim trace from the abstract to a statistical inference in the paper from a stratified random sample of about 30,000 papers from >60 journals from the social-behavioral sciences from 2009 to 2018 with metadata enhancements such as open science badges, links to open access versions of articles, and code availability statements;

2. Confidence scores: Expert and machine ratings of the confidence in Annotation Set claims along with substantial metadata and qualitative assessments about the papers and basis for confidence ratings;

3. Evidence set: A stratified random sample of 600 papers from the Annotation set that additionally assess statistical errors in the papers, process and outcome reproducibility, robustness, and/or replicability;

4. Enhanced bushel set: After 200 of the 600 papers undergo further enhancement by extracting a full bushel of claims tracing from the abstract to statistical inferences in the paper, experts and machines will provide scores and other assessments of all claims, and some additional reproduction, robustness, and replication evidence will be accumulated for multiple claims in those papers;

5. Process data and artifacts from project execution: Substantial data and documentation about the process of conducting this work and the many additional artifacts that are created along the way, sufficient to extend the artifacts and make it a living body of 
research. Cumulatively, SCORE is the most in-depth examination of credibility of research claims in the social and behavioral sciences ever conducted.

All of the data and materials from SCORE that can be shared without violating publisher intellectual property rights or human participant protections will be made publicly accessible after the program is completed. There are many possible research questions that will be possible to advance with these data by interested researchers. Some of the questions that the SCORE team is already investigating include: What is the strength of evidence in original claims? How do experts and machines evaluate the credibility of claims and how does this vary by discipline, time, topic, and methodology? What are observed reproducibility, robustness, and replicability rates in the sample and how do they likewise vary? How well do humans and machines predict replicability, robustness, and reproducibility? How are credibility indicators related to one another?

\section{Conclusion}

SCORE has aspirational objectives to advance scalable tools for credibility assessment, and will generate substantial research artifacts to support scholarly research on human and machine judgment, replicability and reproducibility, and the nature of research claims. This is made possible by SCORE's greatest asset -- the participation of hundreds of researchers across the social and behavioral sciences contributing to claim extraction, credibility assessment, and reproducibility, robustness, and replication studies. This team science project is generating data that would not otherwise be possible (Uhlmann et al., 2019), and will open doors to many novel investigations to assess and enhance research credibility. If nothing else, the program may provide a case example of the potential for team science in tackling many of the most important challenges in social and behavioral research. 


\section{References}

Altmejd, A., Dreber, A., Forsell, E., Huber, J., Imai, T., Johannesson, M., Kirchler, M., Nave, G., \& Camerer, C. (2019). Predicting the replicability of social science lab experiments. PLOS ONE, 14(12), e0225826. https://doi.org/10.1371/journal.pone.0225826

Arkes, H. R., Dawson, N. V., Speroff, T., Harrell Jr, F. E., Alzola, C., Phillips, R., Desbiens, N., Oye, R. K., Knaus, W., \& Connors Jr, A. F. (1995). The covariance decomposition of the probability score and its use in evaluating prognostic estimates. SUPPORT Investigators. Medical Decision Making: An International Journal of the Society for Medical Decision Making, 15(2), 120-131.

Arrow, K. J., Forsythe, R., Gorham, M., Hahn, R., Hanson, R., Ledyard, J. O., Levmore, S., Litan, R., Milgrom, P., \& Nelson, F. D. (2008). The promise of prediction markets. Science-New York Then Washington-, 320(5878), 877.

Botvinik-Nezer, R., Holzmeister, F., Camerer, C. F., Dreber, A., Huber, J., Johannesson, M., Kirchler, M., Iwanir, R., Mumford, J. A., Adcock, R. A., Avesani, P., Baczkowski, B. M., Bajracharya, A., Bakst, L., Ball, S., Barilari, M., Bault, N., Beaton, D., Beitner, J., ... Schonberg, T. (2020). Variability in the analysis of a single neuroimaging dataset by many teams. Nature, 582(7810), 84-88. https://doi.org/10.1038/s41586-020-2314-9

Brier, G. W. (1950). Verification of forecasts expressed in terms of probability. Monthly Weather Review, 78(1), 1-3.

Buolamwini, J., \& Gebru, T. (2018). Gender shades: Intersectional accuracy disparities in commercial gender classification. Conference on Fairness, Accountability and Transparency, 77-91.

Caliskan, A., Bryson, J. J., \& Narayanan, A. (2017). Semantics derived automatically from language corpora contain human-like biases. Science, 356(6334), 183-186. https://doi.org/10.1126/science.aal4230 
Camerer, C. F., Dreber, A., Forsell, E., Ho, T.-H., Huber, J., Johannesson, M., Kirchler, M., Almenberg, J., Altmejd, A., Chan, T., Heikensten, E., Holzmeister, F., Imai, T., Isaksson, S., Nave, G., Pfeiffer, T., Razen, M., \& Wu, H. (2016). Evaluating replicability of laboratory experiments in economics. Science, 351(6280), 1433-1436. https://doi.org/10.1126/science.aaf0918

Camerer, C. F., Dreber, A., Holzmeister, F., Ho, T.-H., Huber, J., Johannesson, M., Kirchler, M., Nave, G., Nosek, B. A., Pfeiffer, T., Altmejd, A., Buttrick, N., Chan, T., Chen, Y., Forsell, E., Gampa, A., Heikensten, E., Hummer, L., Imai, T., ... Wu, H. (2018). Evaluating the replicability of social science experiments in Nature and Science between 2010 and 2015. Nature Human Behaviour, 2(9), 637-644. https://doi.org/10.1038/s41562-018-0399-z

Chang, A. C., \& Li, P. (2015). Is Economics Research Replicable? Sixty Published Papers from Thirteen Journals Say “Usually Not” (SSRN Scholarly Paper ID 2669564). Social Science Research Network. https://doi.org/10.2139/ssrn.2669564

Chen, K.-Y., Fine, L. R., \& Huberman, B. A. (2003). Predicting the future. Information Systems Frontiers, 5(1), 47-61.

Chen, Y., \& Pennock, D. M. (2010). Designing markets for prediction. Al Magazine, 31(4), $42-52$.

Cooke, R. M., Marti, D., \& Mazzuchi, T. (2021). Expert forecasting with and without uncertainty quantification and weighting: What do the data say? International Journal of Forecasting, 37(1), 378-387. https://doi.org/10.1016/j.ijforecast.2020.06.007

Cova, F., Strickland, B., Abatista, A., Allard, A., Andow, J., Attie, M., Beebe, J., Berniūnas, R., Boudesseul, J., Colombo, M., Cushman, F., Diaz, R., N'Djaye Nikolai van Dongen, N., Dranseika, V., Earp, B. D., Torres, A. G., Hannikainen, I., Hernández-Conde, J. V., Hu, W., ... Zhou, X. (2018). Estimating the Reproducibility of Experimental Philosophy. Review of Philosophy and Psychology, 12. https://doi.org/10/gf28qh 
Dasigi, P., Burns, G. A., Hovy, E., \& de Waard, A. (2017). Experiment segmentation in scientific discourse as clause-level structured prediction using recurrent neural networks. ArXiv Preprint ArXiv:1702.05398.

Dreber, A., Pfeiffer, T., Almenberg, J., Isaksson, S., Wilson, B., Chen, Y., Nosek, B. A., \& Johannesson, M. (2015). Using prediction markets to estimate the reproducibility of scientific research. Proceedings of the National Academy of Sciences, 112(50), 15343-15347. https://doi.org/10.1073/pnas.1516179112

Du, M., Liu, N., Song, Q., \& Hu, X. (2018). Towards explanation of dnn-based prediction with guided feature inversion. Proceedings of the 24th ACM SIGKDD International Conference on Knowledge Discovery \& Data Mining, 1358-1367.

Du, M., Liu, N., Yang, F., \& Hu, X. (2021). Learning credible DNNs via incorporating prior knowledge and model local explanation. Knowledge and Information Systems, 63(2), $305-332$.

Ebersole, C. R., Atherton, O. E., Belanger, A. L., Skulborstad, H. M., Allen, J. M., Banks, J. B., Baranski, E., Bernstein, M. J., Bonfiglio, D. B. V., Boucher, L., Brown, E. R., Budiman, N. I., Cairo, A. H., Capaldi, C. A., Chartier, C. R., Chung, J. M., Cicero, D. C., Coleman, J. A., Conway, J. G., ... Nosek, B. A. (2016). Many Labs 3: Evaluating participant pool quality across the academic semester via replication. Journal of Experimental Social Psychology, 67, 68-82. https://doi.org/10.1016/j.jesp.2015.10.012

Ebersole, C. R., Mathur, M. B., Baranski, E., Bart-Plange, D.-J., Buttrick, N. R., Chartier, C. R., Corker, K. S., Corley, M., Hartshorne, J. K., IJzerman, H., Lazarević, L. B., Rabagliati, H., Ropovik, I., Aczel, B., Aeschbach, L. F., Andrighetto, L., Arnal, J. D., Arrow, H., Babincak, P., ... Nosek, B. A. (2020). Many Labs 5: Testing Pre-Data-Collection Peer Review as an Intervention to Increase Replicability. Advances in Methods and Practices in Psychological Science, 3(3), 309-331. https://doi.org/10.1177/2515245920958687

Errington, T. M., lorns, E., Gunn, W., Tan, F. E., Lomax, J., \& Nosek, B. A. (2014). An open 
investigation of the reproducibility of cancer biology research. ELife, 3, e04333. https://doi.org/10.7554/eLife.04333

Forsell, E., Viganola, D., Pfeiffer, T., Almenberg, J., Wilson, B., Chen, Y., Nosek, B. A., Johannesson, M., \& Dreber, A. (2018). Predicting replication outcomes in the Many Labs 2 study. Journal of Economic Psychology, 102117. https://doi.org/10.1016/j.joep.2018.10.009

Forsell, E., Viganola, D., Pfeiffer, T., Almenberg, J., Wilson, B., Chen, Y., Nosek, B. A., Johannesson, M., \& Dreber, A. (2019). Predicting replication outcomes in the Many Labs 2 study. Journal of Economic Psychology, 75, 102117. https://doi.org/10.1016/j.joep.2018.10.009

Forsythe, R., Nelson, F., Neumann, G. R., \& Wright, J. (1992). Anatomy of an experimental political stock market. The American Economic Review, 1142-1161.

Forsythe, R., Rietz, T. A., \& Ross, T. W. (1999). Wishes, expectations and actions: A survey on price formation in election stock markets. Journal of Economic Behavior \& Organization, 39(1), 83-110.

Fraser, H., Bush, M., Wintle, B., Mody, F., Smith, E., Hanea, A., Gould, E., Hemming, V., Hamilton, D., Rumpff, L., Wilkinson, D. P., Pearson, R., Thorn, F. S., Ashton, R., Willcox, A., Gray, C. T., Head, A., Ross, M., Groenewegen, R., ... Fidler, F. (2021). Predicting reliability through structured expert elicitation with repliCATS (Collaborative Assessments for Trustworthy Science). MetaArXiv. https://doi.org/10.31222/osf.io/2pczv

Gibbons, J. D., \& Fielden, J. D. G. (1993). Nonparametric measures of association. Sage. Gordon, M., Viganola, D., Bishop, M., Chen, Y., Dreber, A., Goldfedder, B., Holzmeister, F., Johannesson, M., Liu, Y., Twardy, C., Wang, J., \& Pfeiffer, T. (2020). Are replication rates the same across academic fields? Community forecasts from the DARPA SCORE programme. Royal Society Open Science, 7(7), 200566.

https://doi.org/10.1098/rsos.200566 
Gordon, M., Viganola, D., Dreber, A., Johannesson, M., \& Pfeiffer, T. (2021). Predicting replicability_Analysis of survey and prediction market data from large-scale forecasting projects. ArXiv:2102.00517 [Stat]. http://arxiv.org/abs/2102.00517

Hanea, A., Wilkinson, D. P., McBride, M., Lyon, A., Ravenzwaaij, D. van, Thorn, F. S., Gray, C. T., Mandel, D. R., Willcox, A., Gould, E., Smith, E., Mody, F., Bush, M., Fidler, F., Fraser, H., \& Wintle, B. (2021). Mathematically aggregating experts' predictions of possible futures. MetaArXiv. https://doi.org/10.31222/osf.io/rxmh7

Huber, P., \& Carenini, G. (2019). Predicting discourse structure using distant supervision from sentiment. ArXiv Preprint ArXiv:1910.14176.

Kerr, N. L., \& Tindale, R. S. (2004). Group Performance and Decision Making. Annual Review of Psychology, 55(1), 623-655. https://doi.org/10.1146/annurev.psych.55.090902.142009

Klein, R. A., Ratliff, K. A., Vianello, M., Adams, R. B., Bahník, Š., Bernstein, M. J., Bocian, K., Brandt, M. J., Brooks, B., Brumbaugh, C. C., Cemalcilar, Z., Chandler, J., Cheong, W., Davis, W. E., Devos, T., Eisner, M., Frankowska, N., Furrow, D., Galliani, E. M., ... Nosek, B. A. (2014). Investigating Variation in Replicability: A “Many Labs” Replication Project. Social Psychology, 45(3), 142-152. https://doi.org/10.1027/1864-9335/a000178

Klein, R. A., Vianello, M., Hasselman, F., Adams, B. G., Reginald B. Adams, J., Alper, S., Vega, D., Aveyard, M., Axt, J., \& Babaloia, M. (2018). Many Labs 2: Investigating Variation in Replicability Across Sample and Setting. Advances in Methods and Practice in Psychological Science.

Lanka, S. S. T., Rajtmajer, S. M., \& Giles, C. L. (2021). Extraction and evaluation of statistical information from social and behavioral science papers. Workshop on Scientific Knowledge (Sci-K) at The Web Conference.

Larson, J., Mattu, S., Kirchner, L., \& Angwin, J. (2016). How we analyzed the COMPAS recidivism algorithm. ProPublica (5 2016), 9(1).

Liu, Y., Wang, J., \& Chen, Y. (2020). Surrogate Scoring Rules. Proceedings of the 21st ACM 
Conference on Economics and Computation, 853-871.

https://doi.org/10.1145/3391403.3399488

Malkiel, B. G., \& Fama, E. F. (1970). Efficient capital markets: A review of theory and empirical work. The Journal of Finance, 25(2), 383-417.

McCullough, B. D., McGeary, K. A., \& Harrison, T. D. (2008). Do economics journal archives promote replicable research? Canadian Journal of Economics/Revue Canadienne d'économique, 41(4), 1406-1420. https://doi.org/10.1111/j.1540-5982.2008.00509.x

Mellers, B., Stone, E., Atanasov, P., Rohrbaugh, N., Metz, S. E., Ungar, L., Bishop, M. M., Horowitz, M., Merkle, E., \& Tetlock, P. (2015). The psychology of intelligence analysis: Drivers of prediction accuracy in world politics. Journal of Experimental Psychology: Applied, 21(1), 1-14. https://doi.org/10.1037/xap0000040

Mellers, B., Stone, E., Murray, T., Minster, A., Rohrbaugh, N., Bishop, M., Chen, E., Baker, J., Hou, Y., Horowitz, M., Ungar, L., \& Tetlock, P. (2015). Identifying and Cultivating Superforecasters as a Method of Improving Probabilistic Predictions. Perspectives on Psychological Science, 10(3), 267-281. https://doi.org/10.1177/1745691615577794

Modukuri, S. A., Rajtmajer, S., Squicciarini, A. C., Wu, J., \& Giles, C. L. (2021). Understanding and Predicting Retractions of Published Work.

Nakshatri, N., Menon, A., Giles, C. L., Rajtmajer, S., \& Griffin, C. (2021). Design and Analysis of a Synthetic Prediction Market using Dynamic Convex Sets. ArXiv Preprint ArXiv:2101.01787.

Nosek, B. A., \& Errington, T. M. (2020). What is replication? PLOS Biology, 18(3), e3000691. https://doi.org/10.1371/journal.pbio.3000691

Nosek, B. A., Hardwicke, T. E., Moshontz, H., Allard, A., Corker, K. S., Dreber, A., Fidler, F., Hilgard, J., Kline, M., Nuijten, M. B., Rohrer, J. M., Romero, F., Scheel, A. M., Scherer, L. D., Schönbrodt, F. D., \& Vazire, S. (2021). Replicability, Robustness, and Reproducibility in Psychological Science. Annual Review of Psychology. 
Open Science Collaboration. (2015). Estimating the reproducibility of psychological science. Science, 349(6251), aac4716-aac4716. https://doi.org/10.1126/science.aac4716

Pawel, S., \& Held, L. (2020). Probabilistic forecasting of replication studies. PLOS ONE, 15(4), e0231416. https://doi.org/10.1371/journal.pone.0231416

Pearson, R., Fraser, H., Bush, M., Mody, F., Widjaja, I., Head, A., Wilkinson, D. P., Sinnott, R., Wintle, B., \& Burgman, M. (2021). Eliciting group judgements about replicability: A technical implementation of the IDEA Protocol. Proceedings of the 54th Hawaii International Conference on System Sciences, 461.

Pepe, M. S. (2003). The statistical evaluation of medical tests for classification and prediction. Medicine.

Pfeiffer, T., Chen, Y., Viganola, D., Bishop, M., Dreber, A., Johannesson, M., \& Twardy, C. R. (2020). Prereg Replication Markets Rounds 1-10 (Version 7). Open Science Framework. osf.io/svg3x

Plott, C. R., \& Sunder, S. (1988). Rational expectations and the aggregation of diverse information in laboratory security markets. Econometrica: Journal of the Econometric Society, 1085-1118.

Plott, C. R., Wit, J., \& Yang, W. C. (2003). Parimutuel betting markets as information aggregation devices: Experimental results. Economic Theory, 22(2), 311-351.

Radner, R. (1979). Rational expectations equilibrium: Generic existence and the information revealed by prices. Econometrica: Journal of the Econometric Society, 655-678.

Satopää, V. A., Baron, J., Foster, D. P., Mellers, B. A., Tetlock, P. E., \& Ungar, L. H. (2014). Combining multiple probability predictions using a simple logit model. International Journal of Forecasting, 30(2), 344-356. https://doi.org/10.1016/j.ijforecast.2013.09.009

Silberzahn, R., Uhlmann, E. L., Martin, D. P., Anselmi, P., Aust, F., Awtrey, E., Bahník, Š., Bai, F., Bannard, C., Bonnier, E., Carlsson, R., Cheung, F., Christensen, G., Clay, R., Craig, M. A., Dalla Rosa, A., Dam, L., Evans, M. H., Flores Cervantes, I., ... Nosek, B. A. 
(2018). Many Analysts, One Data Set: Making Transparent How Variations in Analytic Choices Affect Results. Advances in Methods and Practices in Psychological Science, 1(3), 337-356. https://doi.org/10.1177/2515245917747646

Simonsohn, U., Simmons, J. P., \& Nelson, L. D. (2020). Specification curve analysis. Nature Human Behaviour, 1-7. https://doi.org/10.1038/s41562-020-0912-z

Steyvers, M., Wallsten, T. S., Merkle, E. C., \& Turner, B. M. (2014). Evaluating probabilistic forecasts with Bayesian signal detection models. Risk Analysis, 34(3), 435-452.

Uhlmann, E. L., Ebersole, C. R., Chartier, C. R., Errington, T. M., Kidwell, M. C., Lai, C. K., McCarthy, R. J., Riegelman, A., Silberzahn, R., \& Nosek, B. A. (2019). Scientific utopia III: Crowdsourcing science. Perspectives on Psychological Science, 14(5), 711-733.

Wang, G., Kulkarni, S. R., Poor, H. V., \& Osherson, D. N. (2011). Aggregating large sets of probabilistic forecasts by weighted coherent adjustment. Decision Analysis, 8(2), $128-144$.

Wintle, B. C., Fraser, H., Singleton-Thorn, F., Hanea, A. M., Wilkinson, D. P., Bush, M., McBride, M., Gould, E., Head, A., Rumpff, L., \& Fidler, F. (2021). Eliciting reasoning about replicability in social and behavioural sciences.

Wood, B. D. K., Müller, R., \& Brown, A. N. (2018). Push button replication: Is impact evaluation evidence for international development verifiable? PLOS ONE, 13(12), e0209416. https://doi.org/10.1371/journal.pone.0209416

Wu, J., Nivargi, R., Lanka, S. S. T., Menon, A. M., Modukuri, S. A., Nakshatri, N., Wei, X., Wang, Z., Caverlee, J., \& Rajtmajer, S. M. (2021). Predicting the Reproducibility of Social and Behavioral Science Papers Using Supervised Learning Models. ArXiv Preprint ArXiv:2104.04580.

Wu, J., Wang, P., Wei, X., Rajtmajer, S., Giles, C. L., \& Griffin, C. (2020). Acknowledgement Entity Recognition in CORD-19 Papers. Proceedings of the First Workshop on Scholarly Document Processing, 10-19. 
Yang, F., Liu, N., Wang, S., \& Hu, X. (2018). Towards interpretation of recommender systems with sorted explanation paths. 2018 IEEE International Conference on Data Mining (ICDM), 667-676.

Yang, Y., Youyou, W., \& Uzzi, B. (2020). Estimating the deep replicability of scientific findings using human and artificial intelligence. Proceedings of the National Academy of Sciences, 117(20), 10762-10768. https://doi.org/10.1073/pnas.1909046117

Yaniv, I., Yates, J. F., \& Smith, J. K. (1991). Measures of discrimination skill in probabilistic judgment. Psychological Bulletin, 110(3), 611. 


\section{Supplemental Information}

\section{Authors' note}

This work was supported by the Defense Advanced Research Projects Agency. This paper is authored by members of the teams that are directly involved in the SCORE program, but many of the activities - both those funded by the SCORE program itself and the other scientific collaborations that are beginning to form - involve both extensive staff within each team and formal and informal collaborations at other institutions. While this full network of contributors are not authors on this paper, they are critical to the program's execution. Future articles and other scientific contributions resulting from SCORE will be carried out both by large collaborative teams and by the smaller lists of authors that are more typical for many of the fields represented in this program. Co-authors with an affiliation to the Center for Open Science acknowledge a conflict of interest as employees of the nonprofit organization with a mission to increase openness, integrity, and reproducibility of research.

\section{Authors' contributions}

For this paper, contributions are summarized using the following categories:

A. Contributed descriptions of their organizations' specific roles in the program

B. Contributed significantly to the writing of other sections (e.g. introduction and conclusion).

C. Integrated these sections to construct the first draft.

D. Provided feedback and revisions to produce the submitted version of this manuscript.

E. Drafted initial structure and outline of the paper

\begin{tabular}{|l|l|l|l|l|}
\hline $\begin{array}{l}\text { Given } \\
\text { Name }\end{array}$ & Family Name & Institution(s) & Location & Contribution \\
\hline Nazanin & Alipourfard & University of Southern California & Los Angeles, CA & A \\
\hline Beatrix & Arendt & Center for Open Science & Charlottesville, VA USA & D \\
\hline Daniel & Benjamin & University of Southern California & Los Angeles, CA & A \\
\hline Noam & Benkler & SIFT & Minneapolis, MN, USA & A \\
\hline Michael & Bishop & & Ottawa, ON, CANADA & A \\
\hline Mark & Burstein & SIFT & Minneapolis, MN, USA & A \\
\hline Martin & Bush & University of Melbourne & Melbourne, Australia & A, D \\
\hline James & Caverlee & Texas A\&M University & College Station, TX, USA & A \\
\hline Yiling & Chen & Harvard University & Cambridge, MA USA & A \\
\hline Chae & Clark & TwoSix Technologies & Arlington, VA, USA & A \\
\hline Anna & Dreber & Stockholm School of Economics & Stockholm, SWEDEN & A \\
\hline Timothy M. & Errington & Center for Open Science & Charlottesville, VA, USA & A, C, D, E \\
\hline
\end{tabular}




\begin{tabular}{|c|c|c|c|c|}
\hline Fiona & Fidler & University of Melbourne & Melbourne, Australia & A \\
\hline Nicholas & Fox & Center for Open Science & Charlottesville, VA USA & $A, D$ \\
\hline Aaron & Frank & RAND Corporation & Arlington, VA & $A$ \\
\hline Hannah & Fraser & University of Melbourne & Melbourne, Australia & A \\
\hline Scott & Friedman & SIFT & Minneapolis, MN, USA & A \\
\hline Ben & Gelman & TwoSix Technologies & Arlington, VA, USA & A \\
\hline James & Gentile & TwoSix Technologies & Arlington, VA, USA & A \\
\hline C Lee & Giles & $\begin{array}{l}\text { The Pennsylvania State } \\
\text { University }\end{array}$ & State College, PA, USA & A \\
\hline Michael & Gordon & Massey University & Auckland, NEW ZEALAND & A \\
\hline Reed & Gordon-Sarney & TwoSix Technologies & Arlington, VA, USA & A \\
\hline Christopher & Griffin & $\begin{array}{l}\text { The Pennsylvania State } \\
\text { University }\end{array}$ & State College, PA, USA & A \\
\hline Timothy & Gulden & RAND Corporation & Santa Monica, CA & A \\
\hline Krystal & Hahn & Center for Open Science & Charlottesville, VA USA & $D$ \\
\hline Robert & Hartman & The MITRE Corporation & McLean, VA & A \\
\hline Felix & Holzmeister & University of Innsbruck & Innsbruck, AUSTRIA & A \\
\hline Xia & $\mathrm{Hu}$ & Texas A\&M University & College Station, TX, USA & A \\
\hline Magnus & Johannesson & Stockholm School of Economics & Stockholm, SWEDEN & A \\
\hline Lee & Kezar & University of Southern California & Los Angeles, CA & A \\
\hline Melissa & Kline Struhl & Center for Open Science & Charlottesville, VA, USA & $A, B, C, D, E$ \\
\hline Ugur & Kuter & SIFT & Minneapolis, MN, USA & A \\
\hline Anthony & Kwasnica & $\begin{array}{l}\text { The Pennsylvania State } \\
\text { University }\end{array}$ & State College, PA, USA & A \\
\hline Dong-Ho & Lee & University of Southern California & Los Angeles, CA & A \\
\hline Kristina & Lerman & University of Southern California & Los Angeles, CA & A \\
\hline Yang & Liu & $\begin{array}{l}\text { University of California, Santa } \\
\text { Cruz }\end{array}$ & Santa Cruz, CA, USA & A \\
\hline Zach & Loomas & Center for Open Science & Charlottesville, VA USA & C, D \\
\hline Bri & Luis & Center for Open Science & Charlottesville, VA USA & D \\
\hline Ian & Magnusson & SIFT & Minneapolis, MN, USA & A \\
\hline Olivia & Miske & Center for Open Science & Charlottesville, VA USA & C, D \\
\hline Fallon & Mody & University of Melbourne & Melbourne, Australia & A \\
\hline Fred & Morstatter & University of Southern California & Los Angeles, CA & A \\
\hline Brian A. & Nosek & $\begin{array}{l}\text { Center for Open Science; } \\
\text { University of Virginia }\end{array}$ & Charlottesville, VA USA & $A, B, C, D, E$ \\
\hline E. Simon & Parsons & Center for Open Science & Charlottesville, VA USA & $D$ \\
\hline David & Pennock & Rutgers University & New Brunswick, NJ, USA & A \\
\hline Thomas & Pfeiffer & Massey University & Auckland, NEW ZEALAND & A \\
\hline
\end{tabular}




\begin{tabular}{|c|c|c|c|c|}
\hline Haochen & $\mathrm{Pi}$ & University of Southern California & Los Angeles, CA & A \\
\hline Jay & Pujara & University of Southern California & Los Angeles, CA & A \\
\hline Sarah & Rajtmajer & $\begin{array}{l}\text { The Pennsylvania State } \\
\text { University }\end{array}$ & State College, PA, USA & A \\
\hline Xiang & Ren & University of Southern California & Los Angeles, CA & A \\
\hline Abel & Salinas & University of Southern California & Los Angeles, CA & A \\
\hline Ravi & Selvam & University of Southern California & Los Angeles, CA & A \\
\hline Frank & Shipman & Texas A\&M University & College Station, TX, USA & A \\
\hline Priya & Silverstein & $\begin{array}{l}\text { Center for Open Science; Institute } \\
\text { for Globally Distributed Open } \\
\text { Research and Education }\end{array}$ & $\begin{array}{l}\text { Charlottesville, VA, USA; } \\
\text { Preston, UK }\end{array}$ & C, D \\
\hline Amber & Sprenger & The MITRE Corporation & McLean, VA & A \\
\hline Anna & Squicciarini & $\begin{array}{l}\text { The Pennsylvania State } \\
\text { University }\end{array}$ & State College, PA, USA & A \\
\hline Stephen & Stratman & The MITRE Corporation & McLean, VA & A \\
\hline Kexuan & Sun & University of Southern California & Los Angeles, CA & A \\
\hline Saatvik & Tikoo & University of Southern California & Los Angeles, CA & A \\
\hline Charles R. & Twardy & Jacobs / George Mason & Herndon/Fairfax, VA USA & A \\
\hline Andrew & Tyner & Center for Open Science & Charlottesville, VA USA & $A, B$ \\
\hline Domenico & Viganola & World Bank & Washington, DC, USA & A \\
\hline Juntao & Wang & Harvard University & Cambridge, MA, USA & A \\
\hline David & Wilkinson & University of Melbourne & Melbourne, Australia & A \\
\hline Bonnie & Wintle & University of Melbourne & Melbourne, Australia & A \\
\hline Jian & Wu & Old Dominion University & Norfolk, VA, USA & A \\
\hline
\end{tabular}

Supplement Table 1. Journals comprising the Common Task Framework (CTF)

\begin{tabular}{|c|l|}
\hline Discipline & Journals \\
\hline Criminology & $\begin{array}{c}\text { Law and Human Behavior } \\
\text { Criminology }\end{array}$ \\
\hline \multirow{5}{*}{ Economics and Finance } & Experimental Economics \\
& Journal of Labor Economics \\
& The Quarterly Journal of Economics \\
& Journal of Political Economy \\
& Econometrica \\
& The Journal of Finance \\
& Journal of Financial Economics \\
& American Economic Journal: Applied Economics \\
& Review of Financial Studies \\
\hline Education & American Educational Research Journal \\
\hline
\end{tabular}




\begin{tabular}{|c|c|}
\hline & $\begin{array}{l}\text { Exceptional Children } \\
\text { Computers \& Education } \\
\text { Contemporary Educational Psychology } \\
\text { Educational Researcher } \\
\text { Journal of Educational Psychology } \\
\text { Learning and Instruction }\end{array}$ \\
\hline Health & $\begin{array}{l}\text { Psychological Medicine } \\
\text { Health Psychology } \\
\text { Social Science \& Medicine }\end{array}$ \\
\hline Management & $\begin{array}{l}\text { Journal of Business Research } \\
\text { The Leadership Quarterly } \\
\text { Academy of Management Journal } \\
\text { Management Science } \\
\text { Journal of Management } \\
\text { Organization Science }\end{array}$ \\
\hline $\begin{array}{c}\text { Marketing and } \\
\text { Organizational Behavior }\end{array}$ & $\begin{array}{l}\text { Journal of Consumer Research } \\
\text { Journal of the Academy of Marketing Science } \\
\text { Journal of Organizational Behavior } \\
\text { Journal of Marketing } \\
\text { Journal of Marketing Research } \\
\text { Organizational Behavior and Human Decision Processes }\end{array}$ \\
\hline Political Science & $\begin{array}{l}\text { Journal of Experimental Political Science } \\
\text { American Journal of Political Science } \\
\text { American Political Science Review } \\
\text { World Politics } \\
\text { British Journal of Political Science } \\
\text { Journal of Conflict Resolution } \\
\text { Comparative Political Studies } \\
\text { World Development }\end{array}$ \\
\hline Psychology & $\begin{array}{l}\text { Journal of Experimental Social Psychology } \\
\text { Journal of Applied Psychology } \\
\text { Journal of Environmental Psychology } \\
\text { Journal of Personality and Social Psychology } \\
\text { Journal of Experimental Psychology: General } \\
\text { Evolution and Human Behavior } \\
\text { Psychological Science } \\
\text { Cognition } \\
\text { European Journal of Personality } \\
\text { Child Development } \\
\text { Journal of Consulting and Clinical Psychology } \\
\text { Clinical Psychological Science }\end{array}$ \\
\hline Public Administration & $\begin{array}{l}\text { Journal of Public Administration Research and Theory } \\
\text { Public Administration Review }\end{array}$ \\
\hline Sociology & Journal of Marriage and Family \\
\hline
\end{tabular}


American Sociological Review

American Journal of Sociology

Demography

Social Forces

European Sociological Review 